\title{
Evaluating the Effect of JPEG and JPEG2000 on Selected Face Recognition Algorithms
}

\author{
Adebayo Kolawole John \\ Dept. of Computer Science, Southwestern University, Okun-Owa, Ijebu-Ode, Nigeria \\ Collawolley3@yahoo.com \\ Onifade Olufade Williams \\ Dept. of Computer Science, University of Ibadan, Ibadan, Nigeria \\ fadowilly@yahoo.com \\ Adekoya Adewale M. \\ Dept. of Mathematics, Tai Solarin College of Education, Omu-Ijebu, Nigeria \\ adekoya.m@yahoo.com
}

\begin{abstract}
Continuous miniaturization of mobile devices has greatly increased its adoption and use by people in various facets of our lives. This has also increased the popularity of face recognition and image processing. Face recognition is now being employed for security purpose opening up the need for further research in recent time. Image compression becomes useful in cases when images need to be transmitted across networks in a less costly way by increasing data volume while reducing transmission time. This work discusses our findings on image compression and its effect on face recognition systems. We studied and implemented three well known face recognition algorithms and observed their recognition accuracy when gallery / probe images were compressed and/or uncompressed as one would naturally expect. For compression purposes, we adopted the JPEG and JPEG2000 coding standard. The face recognition algorithms studied are PCA, ICA and LDA. As a form of an extensive research, experiments conducted include both in compressed and uncompressed domains where the three algorithms have been exhaustively analyzed. We statistically present the results obtained which showed no significant depreciation in the recognition accuracies.
\end{abstract}

Index Terms - Face recognition; image compression, JPEG, JPEG2000, PCA, ICA, LDA.

\section{INTRODUCTION}

Face recognition has continued to attract widespread acceptance and use recently. Apparently, recognizing human faces has been a challenging problem because of the ethnic diversity of faces and variations caused by expressions, gender, pose, illumination and makeup, this has increased the level of research intensity in this promising field. The steady acceptance and use of face recognition systems is unsurprising as they possess the merits of both high accuracy and low intrusiveness. A very good application area of face recognition is where it has been used to identify or verify the person of a given face in still or video images [1]. In a very simple term, the problem of face recognition can be stated as follows: Given a set of face images labeled with the person's identity (the learning set) and an unlabeled set of face images from the same group of people (the test set), identify each person in the test images from among those in the learning set.

Several face recognition algorithms have been proposed by researchers in order to solve the salient problems faced by face recognition systems; these include Principal Component Analysis, Local Feature Analysis, Linear Discriminant Analysis and Fisher face which are all based on dimensionality reduction. Also neural networks, elastic bunch graph theory, 3D morphable models and multi-resolution analysis are some other techniques usually used, to mention a few. Each of these algorithms has typically overcome the shortcomings of one another, thus extending the application areas for face recognition systems. The important applications of face recognition are in areas of biometrics i.e. computer security and human computer interaction [3].

Majority of the current work done in the field of face recognition have focused on making face recognition systems robust and invariants to light intensities, pose of subjects, expressions on the faces of subjects and other details like background and noise. However, very few works have addressed and concentrated on investigating the effect of image compression on face recognition systems' accuracy. This comes a little bit surprising because most multimedia data transmitted on the networks are nowadays always in compressed form in order to save bandwidth consumed and the time it takes to transmit such data, images (of course) inclusive. The transport of images across communication paths, especially as in a face recognition security surveillance system modeled after a grid-computing paradigm [5] is an expensive process, image compression provides an option for reducing the number of bits in transmission. This, in turn, helps increase the volume of data (live 
feeds images) transferred in space of time along with reducing the cost required. Data compression become increasingly important to most computer networks as the volume of data traffic has begun to exceed their capacity for transmission. Several scenarios exist where image compression seems unavoidable. These include:

- $\quad$ image is taken by some imaging device on site and needs to be transmitted to a distant server for verification/identification;

- $\quad$ image is to be stored on a low-capacity chip to be used for verification/identification

- Thousands (or more) images are to be stored on a server as a set of images of known persons to be used in comparisons when verifying/identifying someone. [4].

This paper investigates the effects of image compression on some face recognition algorithms. The compression method adopted in this work is the JPEG standard encoding format [3], [10], [11], [12] which is based on Huffman coding technique. JPEG (Joint Photographic Experts Group) is an international compression standard for continuous-tone still image, i.e. both grayscale and colored images. This standard is designed to support a wide variety of applications for continuous-tone images. Because of the distinct requirement for each of the applications, the JPEG standard has two basic compression methods i.e. the discrete cosine transform based (DCT) compression used in JPEG and the wavelet-based compression used in JPEG2000. JPEG is a lossy compression algorithm. One of the characteristics that make the algorithm very flexible is that the compression rate can be adjusted. If we compress a lot, more information will be lost, but the resulting image size will be smaller. With a smaller compression rate we obtain a better quality, but the size of the resulting image will be bigger. Thus, compression involves making the coefficients in the quantization matrix bigger when we want more compression, and smaller when we want less compression.

The JPEG compression algorithm is based on two visual effects of the human visual system. First, humans are more sensitive to the luminance than to the chrominance. Second, humans are more sensitive to changes in homogeneous areas, than in areas where there is more variation (higher frequencies). JPEG is the most used format for storing and transmitting images on the Internet. JPEG 2000 (Joint Photographic Experts Group 2000) is a wavelet-based image compression standard. It was created by the Joint Photographic Experts Group committee with the intention of superseding their original discrete cosine transform based. JPEG 2000 has higher compression ratios than JPEG. It does not suffer from the uniform blocks, in other words, it characterize JPEG images with very high compression rates. But it usually makes the image more blurred than JPEG.

Since JPEG and JPEG2000 is selected as our choice compression coder, we are not addressing the case whereby other compression formats are put in place, other compression format like the wavelets [27], VQ [28] and fractal [29][30] based compression have been found to produce fascinating compression results. Huffman coding [26] is based on the frequency of occurrence of a data item (pixel in images). The principle is to use a lower number of bits to encode the data that occurs more frequently. Codes are stored in a Code Book which may be constructed for each image or a set of images. In all cases the code book plus encoded data must be transmitted to enable decoding. In our work, we have studied some face recognition algorithms which were implemented to aid our research. These algorithms are the standard Principal Component Analysis (PCA) technique [13], Independent Component Analysis (ICA) and the Linear Discriminant Analysis (LDA) which are all statistical-based face recognition algorithms.

We conducted exhaustive experiments on three algorithms stated above; using the ORL face database which consists of 40 subjects with ten images each under different light intensities, pose and expression along with the FERET database. For the experiments, images were compressed using four ratios i.e. at a compression quality factor ratio of $15 \%, 25 \%, 40 \%$ and $50 \%$ given different qualities and image size reduction under two compression schemes namely JPEG and JPEG2000. Our goal was to statistically show the variance between recognition achieved in compressed (when either test or training images used for face recognition is compressed) domain and uncompressed domain (where neither test of training image is compressed) under differing algorithms as stated above but without considering other issues like poses of subjects and illumination etc. which has been addressed in several existing literatures.

This work is different from the very few works already done on image compression and face recognition, where the experiments have been conducted in spatial domain and having the images compressed to different bit per pixel levels and also fully decompressing the images prior to the recognition process. Here, compression is achieved using the standard JPEG/JPEG2000 quality factor given from 1 to 100 for varying compression. We have been motivated by the scenario prompted as in the system implemented in [5] where images transmitted across the grid network needs maximum reduction in size through compression as well as retaining the look appeal of the original image before compression to be able to achieve optimal recognition as required by the surveillance system implemented. In our work, some experiments were conducted to investigate the effects of compression on the Implemented face recognition systems' accuracy such that in the first, the probe images were compressed and the images in the training database were uncompressed. In the second experiment, the probe images were uncompressed and the images in the training database were compressed with the given compression ratios. In the third experiment, both the training images and the probe images were compressed and finally, we tested the 
algorithms when both the probe images and the training images were uncompressed in order for us to be able to effectively compare recognition achieved in the earlier experiments as against the last experiment. In all, we were able to strike a balance between optimal compression ratio and effective recognition. It should be noted that this is quite exhaustive as against the former researches where in the experiments conducted, either the probe or the gallery image was compressed. We further give a comprehensive statistical data supporting the experiments conducted.

The remaining parts of the paper are organized thus; in the next section, we present the reviewed literatures and discuss issues on image compression in general. The following section presents the related works where we discussed the works that are similar to ours as regards image compression effects on face recognition. This is followed by the section where we describe our experimental setup and the result obtained. The last section contains our conclusion and recommendations for future works.

\section{STATE OF THE ART}

Several existing face recognition algorithms have been discussed in the literature with varying approaches (i.e. holistic or feature based) and with different accuracy obtained. One important work was carried out in [13] where a system that locates a head of a person and then recognizes the person by comparing characteristics of the face to known individuals was studied. The system is simple to implement, widely used and achieved good recognition. This system projects face images onto a feature space that spans significant variations among known face images. These significant features are called eigenfaces because they are eigenvectors (principal components) of the set of faces. Belhumeur et al. [33] developed an algorithm insensitive to variations in lighting direction and facial expression. They used a pattern classification approach, and considered each pixel in an image as a coordinate in a high-dimensional space. Their method is based on Fisher's Linear Discriminant and is called Fisherface method. In [34], a new algorithm called Discriminant eigenfeatures for face recognition was proposed. It is different to PCA as it preforms eigenvalues analysis on separation matrix. Wiskott and Fellous [35] presented a system for face recognition where faces were described in the form of image graphs. Facial landmarks were described by sets of wavelet components also called jets. Recognition is based on comparison of image graphs. Reference [36] proposed a technique for face recognition based on Bayesian analysis of image differences. In the work presented in [37], a method called Independent Component Analysis was developed, a generalization of PCA, which uses high-order statistics to analyse high-order relationships between pixels. The authors of [38] presented a face recognition system based on combination of Support Vector Machines and Elastic Graph Matching. The authors in [39] proposed a novel face recognition method tor Machines and Elastic Graph Matching. The authors also proposed a novel face recognition method based on dual-tree complex wavelet transform and independent component analysis. Wei et al. [40] developed a technique for face recognition that combines Wavelet transformation, PCA and support vector machine. In [21], Adebayo et al developed a hybrid system based on the well-known PCA blended with a feature based technique which uses some extracted key features in faces for ranking along with the principal components, the system also achieved good recognition.

Compression of images [5], [6], [7], [8], [9] as a research issue has been a subject of research for some time now; this is because the need for an efficient technique for compressing images has increased. Obviously, this stems from the fact that huge amounts of disk space and bandwidth consumption required by images seems to be a big disadvantage during transmission and storage. Images are compressed for different reasons like storing the images in a small memory like mobile devices or low capacity devices, for transmitting the large data over network, or storing large number of images in databases for research purposes. This is important because compressed images occupy less memory space or it can be transmitted faster due to its small size. Based on this reason, the effects of image compression on face recognition systems have gained significant impetus and importance and have become one of the important areas of research work in biometric systems.

As it will be discussed later in this paper, compression can be categorized to lossy and lossless methods. Experiments conducted on face recognition has, however, often used uncompressed images as probes images and training images without taking cognizance that images can be transmitted as probe over the network. Thus, the smaller the size of such images, the higher the images in terms of volumes that can be transmitted and of course, the more effective such systems will be.

\subsection{Image Compression Basic}

Computer images are extremely data intensive and hence require large amounts of memory for storage. As a result, the transmission of an image from one machine to another can be very time consuming. By using data compression techniques, it is possible to remove some of the redundant information contained in images, requiring less storage space and less time to transmit. Compressing an image is significantly different than compressing raw binary data. Of course, general purpose compression programs can be used to compress images, but the result is less than optimal. This is because images have certain statistical properties which can be exploited by encoders specifically designed for them. Also, some of the finer details in the image can be sacrificed for the sake of saving a little more bandwidth or storage space. Compression is achieved by the removal of one or more of the three basic data redundancies which are the coding redundancy, inter- 
pixel redundancy and the psycho-visual redundancy. Coding redundancy is present when less than optimal code words are used. Inter-pixel redundancy results from correlations between the pixels of an image while psycho-visual redundancy is due to data ignored by the human visual system (i.e. visually non- essential information). Image compression techniques reduce the number of bits required to represent an image by taking advantage of these redundancies. An inverse process called decompression (decoding) is applied to the compressed data to get the reconstructed image.

The objective of compression is to reduce the number of bits as much as possible, while keeping the resolution and the visual quality of the reconstructed image as close to the original image as possible. Image compression systems are composed of two distinct structural blocks i.e. an encoder and a decoder as shown in the figure 1 below.

The process in itself is quite simple, an image is fed into the encoder, which creates a set of symbols from the input data and uses them to represent the image. Assuming we represent the number of information carrying units (usually bits) in the original and encoded images respectively with $\mathrm{x}$ and $\mathrm{y}$, then, we can compute the compression ratio, $\mathrm{R}$ by a simple formula.

$\mathrm{R}=\frac{X}{Y}$

As shown in Fig 1 below, the encoder is responsible for reducing the coding, inter-pixel and psycho-visual redundancies of the input image. In the first stage, the mapper transforms the input image into a format designed to reduce inter-pixel redundancies. Then, the quantizer block reduces the accuracy of mapper's output in accordance with a predefined criterion. In the third and final stage, a symbol decoder creates a code for quantizer output and maps the output in accordance with the code. These blocks perform, in reverse order, the inverse operations of the encoder's symbol coder and mapper block. Generally, image compression is classified into two i.e. lossy and lossless compressions [8].

In lossless compression techniques, the original image can be perfectly recovered from the compressed (encoded) image. These are also called noiseless since they do not add noise to the signal (image).It is also known as entropy coding since it uses statistics/decomposition techniques to eliminate/minimize redundancy. Run length encoding, Huffman encoding and LZW coding are examples of techniques under this group. Lossy compression however provides much higher compression ratios than lossless schemes but concedes a certain loss of accuracy in exchange for greatly increased compression. They are widely used since the quality of the reconstructed images is adequate for most applications. Here, the decompressed image is not identical to the original image, but reasonably close to it. Transformation Coding, Vector Quantization, Fractal Coding, Block Truncation Coding and Sub-band Coding are famous lossy techniques. Interested readers can consult references [6] [7] [8] [9] [10] and [11].

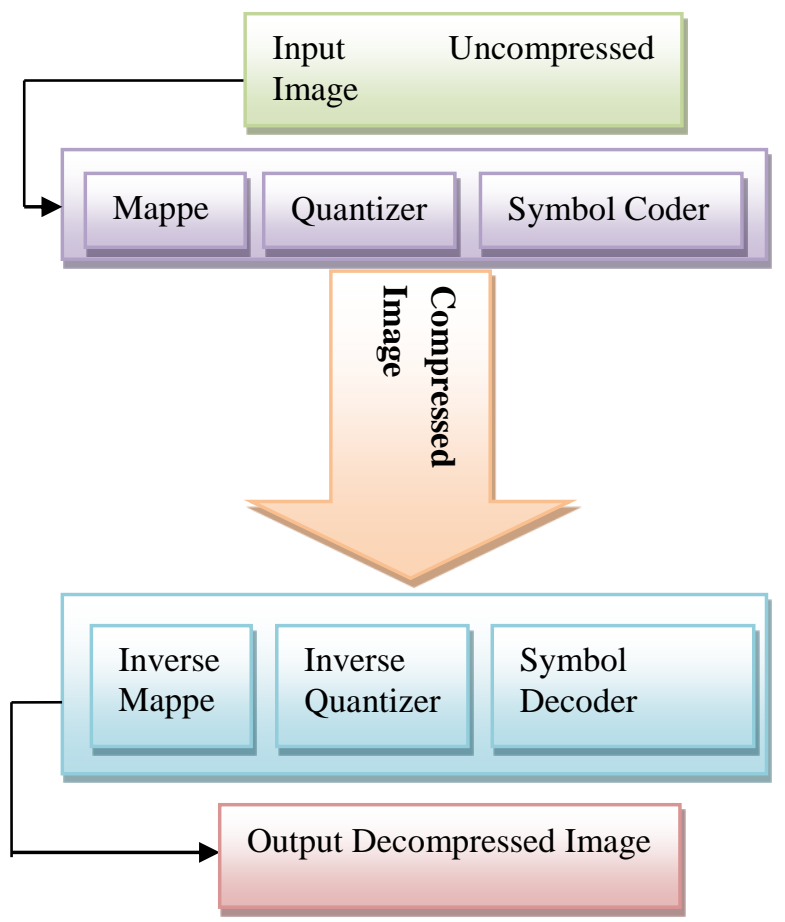

Figure. 1: Image compression process [45]

In this work, JPEG and JPEG2000 standard encoding format is employed in compressing the images. JPEG works by partitioning images into overlapped $8 \times 8$ blocks. A discrete Cosine transform (DCT) [31][32] is applied to each block to convert the gray levels of pixels in the spatial domain into coefficients in the frequency domain. The coefficients are normalized by different scales according to the quantization table provided by the JPEG standard conducted by some psycho visual evidence. The quantized coefficients are rearranged in a zigzag scan order to be further compressed by an efficient lossless coding strategy such as run length coding, arithmetic coding, or Huffman coding. The decoding is simply the inverse process of encoding. So, the JPEG compression takes about the same time for both encoding and decoding. The information loss occurs in the process of coefficient quantization. JPEG as a lossy technique provides best compression rates with complex 24-bit (True Colour) images. It achieves its effect by discarding image data which is imperceptible to the human eye, using DCT as described above which assigns values to different spatial frequency components in the image. It then applies Huffman encoding to achieve further compression. Since the human visual system is less sensitive to higher frequencies, the coefficients representing such frequencies can be discarded, thus yielding higher compression rates. JPEG is irreversible, thus the original image cannot be reconstructed from the compressed file (this is because some coefficients were discarded). JPEG2000 gives a better performance in terms of compression when compared with JPEG because of the 
flexibility of its codestream. It provides both lossy and lossless compression provided by the use of a reversible integer wavelet transform. First, an image is divided into rectangular, non-overlapping tiles on a regular grid. Arbitrary tile sizes are allowed, up to and including the entire image. Components with different subsampling factors are tiled with respect to a high resolution grid, which ensures spatial consistency of the resulting tilecomponents [46]. Each tile of a component must be of the same size; with the exception of tiles around the border (all four sides) of the image. After this transform, all components are treated independently (although different quantization is possible with each component, as well as joint rate allocation across components). Given a tile, wavelet transform is performed. After quantization, each sub-band is subjected to a "packet partition." This packet partition divides each sub-band into regular non-overlapping rectangles. The packet partition provides a medium-grain level of spatial locality in the bit-stream for the purpose of memory efficient implementations, streaming, and (spatial) random access to the bit-stream, at a finer granularity than that provided by tiles [46]. Finally, code-blocks are obtained by dividing each packet partition location into regular non-overlapping rectangles. The code-blocks are then the fundamental entities for the purpose of entropy coding. The next section presents the reviewed existing works on image compression for face recognition as it directly relates with the focus of this work.

\subsection{Related Works}

Kresmic Delac et al [16] analysed the effects that JPEG and JPEG2000 compression standard have on Principal Component Analysis, Linear Discriminant Analysis and Independent Component Analysis. McNemar's hypothesis test was used to compare recognition accuracy. They conducted their experiments using the grayscale portion of the FERET database. They compressed the image using JPEG and JPEG2000 standard with all their experiments done in pixel domain. They compressed the image to $1.0,0.5,0.3$ and 0.2 bits per pixel and then uncompressed prior to recognition process. Their experiments was in two fold i.e. in one, only probe images were compressed and training and gallery images uncompressed while in the other test, compressed images was used for both the training and testing stage. They showed with their result that, compression does not totally reduce performance but even improves it slightly in some cases.

Reference [15] detailed the experiment conducted by Delac et al. Both JPEG and JPEG2000 compression method was used over a wide range of subspace algorithm. They concluded that compression does not affect performance significantly based on McNemar's hypothesis test used. Some performance improvements were also claimed, but none of them were statistically significant. Also, Wijaya et al. in [17] performed face verification on images compressed to 0.5 bit per pixel (bpp) by JPEG2000 and showed that high recognition rates can be achieved using correlation filters. Their conclusion was also that compression does not adversely affect performance. In reference [18], effects of image compression on face recognition systems was investigated. They compressed the probe images with JPEG while the training images were uncompressed. Their experiment was conducted using the FERET database and its dup1probe set. Images were compressed to $0.8,0.4,0.25$ and $0.2 \mathrm{bpp}$. They claimed that compression does not affect recognition significantly across wide range of compression rates but noted a drop in recognition rate at $0.2 \mathrm{bpp}$ and below.

Reference [19] tested the effects of standard JPEG compression and a variant of wavelet compression with a PCA+L1 method. Probe images were in both cases compressed to $0.5 \mathrm{bpp}$. The training set of images was uncompressed. FERET database was used along with its standard probe sets. Their results showed no performance drop for JPEG compression and a slight increase for wavelet compression. A work on JPEG2000 was done in [20]. Compression was done at a ratio of 10:1. A commercial face recognition system was used for testing a vendor database. They also claimed no significant performance drop when using compressed probe images. In reference [45], we also analysed image compression effects on three well known face recognition algorithms including PCA, MPCA and MPCALDA. Compression was done based on some quality factor ranging between 15 and 50 . We carried out four separate experiments including three in compressed domain when either the gallery or probe images or both are compressed and one experiment in uncompressed domain in which neither of the gallery or probe images were compressed. A statistical recommendation of the results of the experiments was given.

It can be observed from the reviewed literatures that the few works had only concentrated on measuring the recognition accuracy only when either of the probe or the gallery image has been compressed. We then build on the existing works by investigating the effects of JPEG and JPEG2000 compression on three well known algorithms selected. Inspired and motivated by the work in [16], we extended the experimental domain of that work by extensively studying the slight variations observed in new experiments introduced for the compressed domain. We compared how each of the techniques fared better than the others. To know the compression ratio achieved based on the quality factor used, we use this simple formula:

$\mathrm{R}=\frac{\text { Image Width } * \text { Image Height } * \text { Bit Height }}{\text { Bit per pixel } * \text { File Size }}$

Where $\mathrm{R}$ is the ratio

The following section gives our methodology, experiments conducted and the results obtained. 


\section{RESEARCH DESIGN AND METHODOLOGY}

This research work typically analyses the effect that compression of images has on the recognition accuracy of face recognition systems. Our result was concluded based on the thorough analysis of different experimental setup used to actually investigate our claim. Face recognition system based on PCA, ICA and LDA were used. However, unlike the conventional face recognition experimental setup where images used as training images and/or probe images are in uncompressed domain i.e. not compressed for any sake, we divided our experimental setup into two, the first case analysis is based on using images that are uncompressed as training database members and presenting the result obtained on three different algorithms, the second is based on images compressed using different compression ratio as an incremental factor expressed in form of percentages, the results obtained were then statistically compared to observe any notable shift in the recognition rates recorded for each of the algorithms used in uncompressed domain. This method is most suitable because we can actually track any significant shift in recognition rate when the images are compressed as against when not compressed which gives us a basis for our conclusion. In this work, we measured image quality using peak signal-to-noise ratio (PSNR) [5] as most common objective measure, which does not correlate well with subjective quality measure, and mean-square error [11], which incorporates model of Human Visual System (HVS) and leads to better correlation with the response of the human observers.

The peak to signal ratio is measured by the formula:

$\mathrm{PSNR}=20 \log \frac{2^{n}-1}{R M S}$

Given that

$\mathrm{RMS}=\sqrt{\frac{i}{N} \sum_{i=1}^{N}\left(A_{i}-A_{i}^{\prime}\right)^{2}}$

Below, we present a short review of these three algorithms and the basic steps carried out in implementing these face recognition systems.

\subsection{Principal Component Analysis (PCA)}

Principal component analysis [13], [21] provides a method to efficiently represent a collection of sample points, reducing the dimensionality of the description by projecting the points onto the principal axes, an orthornormal set of axes pointing in the directions of maximum covariance in the data. It minimizes the mean square error for a given number of dimensions and provides a measure of importance for each axis. The algorithm is as follows:
1. Let's assume the face images in our database is $x_{1}$, $\mathrm{x}_{2}, \mathrm{x}_{3}, \mathrm{x}_{4} \ldots \ldots, \mathrm{x}_{\mathrm{M}}$ then we find the mean image which is

$$
\Psi=\frac{1}{m} \sum_{n=1}^{m} X_{n}
$$

2. Next, we have to know how each face differs from the mean image above like this

$$
\phi_{i}=X_{i}-\Psi
$$

This set of very large vectors is then subjected to principal component analysis, which seeks a set of $\mathrm{M}$ orthogonal vectors, Un, which best describes the distribution of the data. The kth vector, UK, is chosen such that the eigenvalues

$\lambda_{k}=\sum_{n=1}^{m}\left(U_{K}^{T} \phi_{n}\right)^{2}$

which is also subject to eigenvector $\mathrm{U}_{\mathrm{I}}^{\mathrm{T}} \mathrm{U}_{\mathrm{K}}$, where the vectors $\mathrm{UK}$ and scalars $\lambda \mathrm{K}$ are the eigenvectors and eigenvalues, respectively of the covariance matrix $\mathrm{C}$ of the training images depicted as

$\mathrm{C}=\frac{1}{m} \sum_{n=1}^{m} \phi_{n} \phi_{n}^{T}=A A^{T}$.

In essence we are calculating the covariance matrix $\mathrm{C}$.

3. The matrix $A=\left[\phi_{1}, \phi_{2}, \phi_{3} \ldots \ldots \phi_{m}\right]$.

The covariance matrix $\mathrm{C}$, however is $\mathrm{N} 2 \times \mathrm{N} 2$ real symmetric matrix, and determining the $\mathrm{N} 2$ eigenvectors and eigenvalues is an intractable task for typical image sizes. We need a computationally feasible method to find these eigenvectors. Following these analyses, we construct the $\mathrm{M} \times \mathrm{M}$ matrix $\mathrm{L}=\mathrm{ATA}$ where

$\mathrm{L}_{\mathrm{mn}}=\phi_{\mathrm{m}}^{\mathrm{t}} \phi_{\mathrm{n}}$

and then find the $\mathrm{M}$ eigenvectors, $\mathrm{V}_{\mathrm{i}}$ of $\mathrm{L}$. These vectors determine linear combinations of the $\mathrm{M}$ training set of face images to form the eigenfaces $U_{i}$. Which we represent as

$\mathrm{U}_{\mathrm{I}}=\sum_{k=1}^{m} V_{I K} \phi_{K}$

where $\mathrm{I}=1 \ldots . . \mathrm{M}$. The associated eigenvalues allow us to rank the eigenvectors based on how useful they are in characterizing the variation among the images.

\subsection{Independent Component Analysis (ICA)}

While PCA decorrelates the input data using secondorder statistics and thereby generates compressed data with minimum mean-squared re-projection error, ICA minimizes both second-order and higher-order dependencies in the input. It is intimately related to the blind source separation (BSS) problem, where the goal is to decompose an observed signal into a linear 
combination of unknown independent signals. In this paper, we use the Fast ICA algorithm [37] as against other methods such as InfoMax or Maximum likelihood [37] that can also be employed.

The FastICA method computes independent components by maximizing non-Gaussianity of whitened data distribution using a kurtosis maximization process [48]. The kurtosis measures the non-Gaussianity and the sparseness of the face representations.

Let $S$ be the vector of unknown source signals and $x$ be the vector of observed mixtures. If $A$ is the unknown mixing matrix, then the mixing model is written as

$\mathrm{x}=\mathrm{As}$

It is assumed that the source signals are independent of each other and the mixing matrix $\mathrm{A}$ is invertible. Based on these assumptions and the observed mixtures, ICA algorithms try to find the mixing matrix A or the separating matrix $\mathrm{W}[41]$ such that

$\mathrm{U}=\mathrm{Wx}=\mathrm{WAs}$

is an estimation of the independent source signals.

Independent Component Analysis aims to transform the data as linear combinations of statistically independent data points. Therefore, its goal is to provide an independent rather that uncorrelated image representation. ICA [42] is an alternative to PCA which provides a more powerful data representation. It's a discriminant analysis criterion, which can be used to enhance PCA. The ICA algorithm is briefly detailed below:

Let $c x$ be the covariance matrix of an image sample $\mathrm{X}$. The ICA of $\mathrm{X}$ factorizes the covariance matrix $\mathrm{C}_{\mathrm{x}}$ into the following form

$\mathrm{C}_{\mathrm{x}}=\mathrm{F} \Delta \mathrm{F}^{\mathrm{T}}$

where $\Delta$ is diagonal real positive and $F$ transforms the original data into $\mathrm{Z}(\mathrm{X}=\mathrm{FZ})$. The components of $\mathrm{Z}$ will be the most independent possible. To derive the ICA transformation $\mathrm{F}$,

$\mathrm{X}=\Phi \Lambda^{1 / 2} \mathrm{U}$

Where $\mathrm{X}$ and $\Lambda$ are derived solving the following Eigen problem:

$\mathrm{C}_{\mathrm{x}}=\Phi \Lambda \Phi^{\mathrm{T}}$

Then, there are rotation operations which derive independent components minimizing mutual information. Finally, normalization is carried out.

\subsection{Linear Discriminant Analysis (LDA)}

LDA is widely used to find linear combinations of features while preserving class separability. Unlike PCA, LDA tries to model the differences between classes.
Classic LDA is designed to take into account only two classes. Specifically, it requires data points for different classes to be far from each other, while points from the same class are close. Consequently, LDA obtains differenced projection vectors for each class. Multi-class LDA algorithms which can manage more than two classes are more used.

Suppose we have $\mathrm{m}$ samples $\mathrm{x} 1, \ldots, \mathrm{X}_{\mathrm{m}}$ belonging to $\mathrm{c}$ classes; each class has $M_{k}$ elements. We assume that the mean has been extracted from the samples, as in PCA. The objective function of the LDA [44] can be defined as

$a_{\text {opt }}=\operatorname{argmax} \frac{a^{T} S_{b} a}{a^{T} S_{t} a}$

$S_{b}=\sum_{k=1}^{c} m_{k} \mu^{(k)}\left(\mu^{(k)}\right)^{T}=$

$\sum_{k=1}^{c}\left\{\left(\frac{1}{m_{k}}\left(\sum_{i=1}^{m_{k}} x_{i}^{(k)}\right)\right)\left(\frac{1}{l_{k}}\left(\sum_{i=1}^{m_{k}} x_{i}^{(k)}\right)\right)\right\}^{T}=$

$X W_{m x m} X^{T}$

$S_{t}=\sum_{i=1}^{m} x_{i}\left(x_{i}\right)^{T}=X X^{T}$

Where $W_{m x m}$ is a diagonal matrix defined as

$$
W_{m x m}=\left[\begin{array}{cccc}
W^{1} & 0 & \ldots & 0 \\
0 & W^{2} & \ldots & 0 \\
\vdots & \vdots & \ddots & \vdots \\
0 & 0 & \ldots & W^{c}
\end{array}\right]
$$

And $W^{k}$ is a $m_{k} \mathrm{X} m_{k}$ matrix.

$$
W^{k}=\left[\begin{array}{cccc}
\frac{1}{m_{k}} & \frac{1}{m_{k}} & \cdots & \frac{1}{m_{k}} \\
\frac{1}{m_{k}} & \frac{1}{m_{k}} & \cdots & \frac{1}{m_{k}} \\
\vdots & \vdots & \ddots & \vdots \\
\frac{1}{m_{k}} & \frac{1}{m_{k}} & \cdots & \frac{1}{m_{k}}
\end{array}\right]
$$

The eigenproblem can then finally be written as:

$S_{b} a=\lambda S_{t} a \rightarrow S_{t}^{-1}$

$S_{b} a=\lambda \mathrm{a} \rightarrow \mathrm{X} W_{l x l} X^{T}\left(\mathrm{X} X^{T}\right)^{-1} \mathrm{a}=\lambda \mathrm{a}$

The solution of this eigenproblem provides the eigenvectors. The embedding is however done as done in PCA algorithm

We have quickly described the algorithms adopted for our work. The following section describes the experimental setup in terms of the database used and some other necessary details.

\section{EXPERIMENTAL SETUP AND PROCEDURE}

\subsection{Database Used}

We adopted two of the foremost face databases i.e. the ORL and FERET databases. The ORL face database consists of 40 subjects with 10 images per subject. The images were taken under different pose, light intensities and facial expressions. The dimension of each image is 
92 by 112 in which the background has been typically removed.

On the other hand, The FERET face recognition database is a set of face images collected by NIST from 1993 to 1997. The FERET database contains images of 1,196 individuals, with up to 5 different images captured for each individual. The images are separated into two sets i.e. the gallery images and the probe/test images.

Each image contains a single face. Prior to processing, the faces are registered to each other, and the backgrounds are eliminated. In this study, only head-on images are used; faces in profile or at other angles are discarded. Of particular interest is the structure of the database. The gallery contains 1,196 face images. For this study, the training images are a randomly selected subset of 500 gallery images. Most importantly, there are four different sets of probe images: using the terminology in [12], the fafb probe set contains 1,195 images of subjects taken at the same time as the gallery images. The only difference is that the subjects were told to assume a different facial expression than in the gallery image. The duplicate I probe set contains 722 images of subjects taken between one minute and 1,031 days after the gallery image was taken. The duplicate II probe set is a subset of the duplicate I probe set, where the probe image is taken at least 18 months after the gallery image. The duplicate II set has 234 images. Finally, the fafc probe set contains images of subjects under significantly different lighting. This is the hardest probe set, but unfortunately it contains only 194 probe images. Gallery images are images with known labels, while probe images are matched to gallery images for identification.

The database is summarized as briefed below:

1) FB: Two images were taken of an individual, one after the other. In one image, the individual has a neutral facial expression, while in the other they have non-neutral expressions. One of the images is placed into the gallery file while the other is used as a probe. In this category, the gallery contains 1,196 images and the probe set has 1,195 images.

2) Duplicate I: The only restriction of this category is that the gallery and probe images are different. The images could have been taken on the same day or a year apart. In this category, the gallery consists of the same 1,196 images just like the FB gallery while the probe set contains 722 images.

Fc: Images in the probe set are taken with a different camera and under different lighting than the images in the gallery set. The gallery contains the same 1196 images as the FB and Duplicate I galleries, while the probe set contains 194 images.

3) Duplicate II: Images in the probe set were taken at least 1 year after the images in the gallery. The gallery contains 864 images, while the probe set has 234 images.
For our experiment, we used only the Duplicate II gallery images.

\subsection{Pre-processing}

Histogram equalization [23] was applied on all images prior to the beginning of the experiments. All images were rescaled to 60 by 80 dimensions. Also, the background details were removed.

\subsection{Statistical Approach}

For the experiments conducted using PRL face database, the training database consists of 7 images each of a subject while the 3 remaining images were used as probes. For the FERET database, we randomly select images of 15 subjects from Dup II gallery set. We measured the recognition accuracy obtained based on the number of true positive recognition achieved for each subject when the probes are presented, this was done for the ORL database too. The accuracy is presented in form of percentage showing the number of time the system accurately identified a true individual from the gallery with the image used as probe. The algorithms used as stated earlier are the PCA, ICA and LDA.

\subsection{Experiments}

We present the extensive experiments conducted in this work. We implemented the three algorithms above in MATLAB 2009 on an Intel ICore 3 system with 4GB memory and running at $3 \mathrm{GHz}$ speed. We used images from the ORL database which consists of 10 images per subject out of which 7 images were used in the training of the implemented algorithms and 3 used as probe images for each of the experiments. Also used is the FERET face database which comprises of a larger dataset compared to ORL out of which the DupII gallery set is used in our experiments. Histogram equalization was applied on all the images as said earlier in order to improve recognition accuracy. We avoided the issue of varying the thresholds or the number of images trained per subject for each of the algorithms because this has been done in one of our earlier works and since we are only concerned with significance of compression to recognition accuracy when the algorithms employed are in use.

As said earlier, in expanding the frontier of former researches, the experiments conducted here are in four phases. In the first experiment, only the probe images were compressed while the gallery images were left uncompressed, the reverse of this is done in the second test where the gallery is compressed and the probe images uncompressed. The next experiment involves both the probes and the gallery images being compressed and finally when they are both uncompressed, this we termed "Normal" as obtainable in common face recognition systems evaluation. All the experiments were conducted differently for each of the algorithms employed. Two compression schemes were put to use as explained early in the paper i.e. the JPEG and JPEG2000 coding standard. Based on the 
recognition accuracy recorded under each test for each algorithm, we give our conclusion on the effects generated by the compression done i.e. we want to know if the compression really and/or significantly reduce the recognition accuracy. Also performance evaluation of the algorithms is analyzed respectively in order to determine the best performing algorithm when working in a compressed domain, with this, we also present our observations. The compression technique employed is the JPEG standard encoding format. The compression quality factor used is in ratios $15,25,40$ and 50 , where the ratio is a factor of JPEG encoding quality. The diagrams below describe each of the experiments conducted.

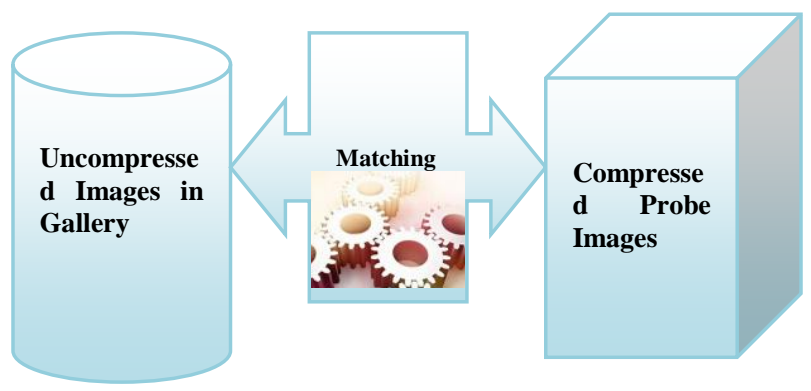

Figure 2: Schematic Representation of Experiment 1



Figure 3: Schematic Representation of Experiment 2

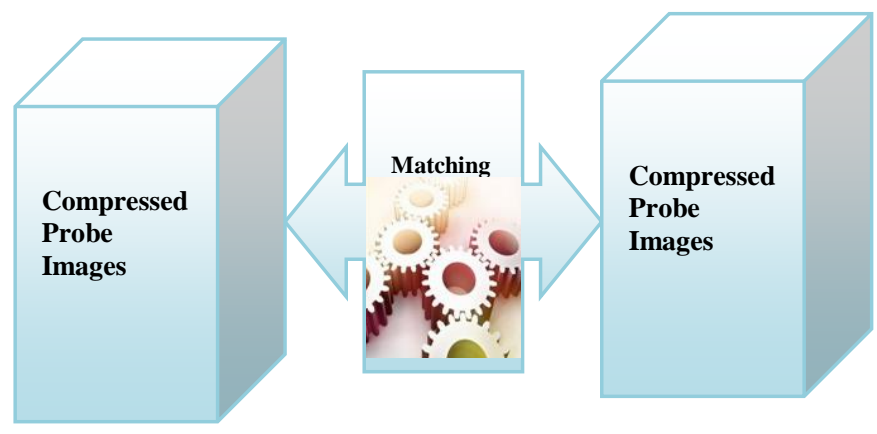

Figure 4: Schematic Representation of Experiment 3

\section{RESULTS}

This section presents the results obtained for the recognition rate on the three algorithms used for the experiments. The tables and charts below summarize the result obtained after conducting the experiments. We take the experiment tagged 'Normal' to be the fourth experiment in which the probes and the training images are not in compressed form as obtainable in standard face recognition experiments. The recognition rate for the experiment tagged "Normal" remains invariant for experiments conducted under each algorithm. The tables below describe the recognition rate achieved under different algorithms. Figures 5, 6 and 7 shows the result obtained when PCA, ICA and LDA respectively was employed with the ORL face database in use. The results obtained with the FERET database with the three algorithms follow suits. follo figure 6 shows when ICA was employed and lastly figure 7 shows when LDA was used. In each of the tables, the column named $15 \%, 25 \%$, $40 \%$ and $50 \%$ shows the incremental degree of compression employed as a quality factor such that an image of compression ratio $50 \%$ is of better quality to an image of compression ratio $10 \%$ etc. The description of experiment is as shown in figures 2, 3 and 4. The only exception is column named 'normal' under each table which shows the result obtained by each algorithm when both the training images and the probe images are left uncompressed as explained earlier. The chart that follows gives a statistical representation of the accuracy of each algorithm under different compression quality factor.

TABLE 1: Experimental results for ORL face database

\begin{tabular}{|l|l|l|l|l|l|l|l|}
\hline EXP & ENC & ALG & NOR & $15 \%$ & $25 \%$ & $40 \%$ & $50 \%$ \\
\hline 1 & JPEG & PCA & 89.9 & 79.3 & 84.0 & 85.2 & 86.5 \\
\hline 2 & JPEG & PCA & 89.9 & 82.4 & 84.7 & 86.1 & 87.0 \\
\hline 3 & JPEG & PCA & 89.9 & 79.2 & 83.7 & 85.0 & 85.1 \\
\hline 1 & JP2K & PCA & 89.9 & 79.3 & 84.2 & 85.3 & 86.9 \\
\hline 2 & JP2K & PCA & 89.9 & 82.5 & 85.0 & 86.2 & 87.1 \\
\hline 3 & JP2K & PCA & 89.9 & 79.2 & 83.9 & 85.1 & 85.5 \\
\hline 1 & JPEG & ICA & 93.2 & 79.3 & 84.1 & 85.2 & 86.6 \\
\hline 2 & JPEG & ICA & 93.2 & 82.6 & 84.7 & 86.4 & 87.0 \\
\hline 3 & JPEG & ICA & 93.2 & 79.7 & 84.0 & 85.1 & 85.2 \\
\hline 1 & JP2K & ICA & 93.2 & 79.3 & 84.2 & 85.6 & 87.0 \\
\hline 2 & JP2K & ICA & 93.2 & 82.8 & 84.5 & 86.4 & 87.1 \\
\hline 3 & JP2K & ICA & 93.2 & 82.7 & 84.8 & 86.9 & 87.8 \\
\hline 1 & JPEG & LDA & 74.6 & 73.0 & 73.6 & 73.9 & 74.7 \\
\hline 2 & JPEG & LDA & 74.6 & 73.3 & 73.7 & 73.9 & 74.9 \\
\hline 3 & JPEG & LDA & 74.6 & 75.2 & 75.4 & 75.4 & 76.5 \\
\hline 1 & JP2K & LDA & 74.6 & 73.1 & 73.6 & 74.1 & 75.0 \\
\hline 2 & JP2K & LDA & 74.6 & 73.5 & 73.8 & 74.3 & 75.4 \\
\hline 3 & JP2K & LDA & 74.6 & 72.3 & 73.1 & 73.7 & 74.0 \\
\hline
\end{tabular}


TABLE 2: Experimental results for DUP II dataset of FERET face database

\begin{tabular}{|l|l|l|l|l|l|l|l|}
\hline EXP & ENC & ALG & NOR & $15 \%$ & $25 \%$ & $40 \%$ & $50 \%$ \\
\hline 1 & JPEG & PCA & 74.3 & 71.1 & 71.5 & 71.8 & 72.5 \\
\hline 2 & JPEG & PCA & 74.3 & 71.3 & 71.5 & 72.0 & 72.5 \\
\hline 3 & JPEG & PCA & 74.3 & 70.4 & 70.6 & 71.0 & 71.3 \\
\hline 1 & JP2K & PCA & 74.3 & 71.4 & 71.5 & 72.3 & 72.9 \\
\hline 2 & JP2K & PCA & 74.3 & 72.5 & 72.7 & 73.2 & 73.9 \\
\hline 3 & JP2K & PCA & 74.3 & 70.6 & 70.7 & 71.3 & 71.3 \\
\hline 1 & JPG & ICA & 83.2 & 82.1 & 82.4 & 82.5 & 84.0 \\
\hline 2 & JPEG & ICA & 83.2 & 82.3 & 82.7 & 82.9 & 83.0 \\
\hline 3 & JPEG & ICA & 83.2 & 82.3 & 82.7 & 82.9 & 83.0 \\
\hline 1 & JP2K & ICA & 83.2 & 82.3 & 82.7 & 82.8 & 82.9 \\
\hline 2 & JP2K & ICA & 83.2 & 82.8 & 84.5 & 86.4 & 87.1 \\
\hline 3 & JP2K & ICA & 83.2 & 82.7 & 82.7 & 82.8 & 83.0 \\
\hline 1 & JPEG & LDA & 72.0 & 67.3 & 67.5 & 67.5 & 68.0 \\
\hline 2 & JPEG & LDA & 72.0 & 68.1 & 68.1 & 68.4 & 68.7 \\
\hline 3 & JPEG & LDA & 72.0 & 67.5 & 67.7 & 67.8 & 68.7 \\
\hline 1 & JP2K & LDA & 72.0 & 68.1 & 68.3 & 68.7 & 69.2 \\
\hline 2 & JP2K & LDA & 72.0 & 70.6 & 70.6 & 70.9 & 71.5 \\
\hline 3 & JP2K & LDA & 72.0 & 71.2 & 71.5 & 71.9 & 71.9 \\
\hline
\end{tabular}

The tables above show the result of recognition in compressed domain under each of the three explored algorithm. The column named 'EXP' gives the type of experiment i.e. is it experiment 1 or experiment 2 etc. based on the initial description of each experiment earlier in the paper. The column named ' $E N C$ ' gives the encoder employed i.e. whether it is JPEG or JPEG2000. We represented JPEG2000 with 'JP2K' and it should be noted that we referred to JPEG2000. The column named 'ALG' gives the algorithm used while the other column shows either the experiment conducted in totally uncompressed domain (termed NOR for normal) or the compression degree expressed in form of quality factor of compression for each image.

Critically looking at the tables above, it can be seen that the recognition accuracy of the three algorithms did not really deteriorate when working with compressed images, however, a slight difference is noted from the recognition rate achieved when both the probe images and the training images are uncompressed (i.e. experiment 4 termed normal) compared with the recognition rate achieved in other experiments. This is however not so significant as one would have expected due to information lost during compression. It is also acceptable bearing in mind the percentage of data discarded during compression.

A critical look also shows that the algorithm's performance was worst when both the probe and the training images are compressed but is also acceptable. The ICA seems to give a better performance than PCA and the LDA generally but nonetheless, the recognition achieved by the PCA is also commendable. Also, the three algorithms were at their lowest ebb in all the experiments for the $15 \%$ quality factor and performed best when working with $50 \%$ quality factor. This lends credence to the fact that the more the information that is lost in an image, the lower the recognition rate that will be achieved. JPEG2000 have been claimed to perform better than its JPEG coding counterpart. This is totally apparent from the tables above; the recognition accuracy recorded for each of the algorithm appears to be slightly better when JPEG2000 is used than when JPEG is used.

\section{CONCLUSION}

This work comparatively analyzed the effect of compression on three well known face recognition algorithms. We have employed the use of two popular encoders namely JPEG and JPEG2000 for compressing images. Experiments have been carried out to investigate how compression of images can affect recognition accuracies. Results obtained have been given with respect to each algorithm used and of course, the compression encoders.

It can be argued from the result that compression effect on recognition accuracy is not too significant. Initially, we thought of experiencing a marginal shift of $+/-15$ when comparing experiments in compressed and uncompressed domain, however, the result obtained totally differs from our expectation. In fact, the significance cannot be compared to the effect that issues like pose (in varying degrees), background noise and illumination have on face recognition accuracies. Thus, we can conclude that while compression does reduce recognition accuracies in the systems tested, the merits of compression in some applications that may rely on effective compression to improve efficiency far outweighs the insignificant difference noted. This will really be of help in systems such as face recognitionbased surveillance systems which employs gridcomputing [5] in its operations where images will need to be compressed and transmitted several times across the grid-networks at a very fast rate. Future works can explore more scenarios where several compression techniques are employed, such as using some lossy and lossless algorithms and comparing how several face recognition algorithms performs under them.

\section{REFERENCES}

[1] M. S. Anish Kumar, Rajesh Cherian Roy, R. Gopikakumari, " A New Image Compression and Decompression technique based on 8x8 MRT", GVIP Journal, Volume 6, Issue 1, July 2006.

[2] M. Kutila, J. Virtanen, "Parallel Image Compression and Analysis with Wavelets", World academy of science, engineering and technology 2005.

[3] Sonal, Dinesh Kumar, " a study of various image 
compression Techniques", Department of Computer Science \& Engineering Guru Jhambheswar University of Science and Technology, Hisar. 2008.

[4] Dinesh Kumar, C.S Rai and Shakti Kumar, "Principal W. Zhao, R. Chellapa, and P. J Phillips, "Face Recognition: A literature survey," in Technical Report, University of, Maryland, 2000.

[5] Adebayo K.J and Onifade O.F.W, "Framework for a Dynamic Grid-Based Surveillance Face Recognition System" In Africa journal of computing and ICT (IEEE Nigerian Section).Pg. 1 - 10, Vol. 4 N0. 1, June, 2011.

[6] D. Kresmir, G. Mislav, and L. Panos, “Appearance Based statistical method for face recognition" in $47^{\text {th }}$ international symposium, ELMAR 2005, Zedar, Croatia. June 2005.

[7] Kresimir Delac, Sonja Grgic and Mislav Grgic, "Image Compression in Face Recognition - a Literature Survey" in recent advances in face recognition, pg236, I-Tech publishers, Vienna, Austria, Dec 2008.

[8] Sonja Grgic, Marta Mrak and Mislav Grgic, "Comparison of jpeg image coders" in recent advances in face recognition, pg236, I-Tech publishers, Vienna, Austria, Dec 2008.

[9] Component Analysis for Data Compression and Face Recognition". 2008.

[10] Bernie Brower, "Image Compression Basics", NIMA Systems engineering service, 2008.

[11] Arcangelo Bruna, "Principles of Image Compression", Advanced System Technology, Catania, 2008.

[12] Rahul Garg and Varun Gulshan, "JPEG Image Compression" Lecture note, Dec 2005.

[13] M. Turk, and A. Pent land., "Eigenfaces for recognition", Journal of Cognitive Neuroscience, Vol. 3, pp. 71-86, (1991).

[14] Haiping Lu, K.N. Plataniotis, and A.N. Venetsanopoulos., "MPCA: Multilinker Principal Component Analysis of Tensor Objects", IEEE Trans on Neural Networks, Vol. 19, No. 1, Page: 18-39, January 2008.

[15] Delac, K.; Grgic, M. \& Grgic, S. "Effects of JPEG and JPEG2000 Compression on Face Recognition", Lecture Notes in Computer Science, Pattern Recognition and Image Analysis, Vol. 3687, 2005, pp. 136-145, 2005.

[16] Delac, K.; Grgic, M. \& Grgic, S., "Image Compression Effects in Face Recognition Systems". In Face Recognition, Book edited by: K Delac and M.Grgic, ISBN 978-3-902613-03-5, pp.558, I-Tech, Vienna, Austria, June2007.

[17] Wijaya, S.L.; Savvides, M. \& Vijaya Kumar B.V.K, "Illumination-Tolerant Face Verification of LowBit-Rate JPEG2000 Wavelet Images with Advanced Correlation Filters for Handheld Devices". In Journal of Applied Optics, Vol. 44, 2005, pp. 655-665, 2005.
[18] Blackburn, D.M.; Bone, J.M. \& Phillips, P.J, "FRVT 2000 Evaluation Report". www.cse.msu.edu/ cse891/sect601/casestudy/eval uationoffacercgsystems.pdf 2001.

[19] Moon, H. \& Phillips, P.J, "Computational and Performance aspects of PCA-based Face Recognition Algorithms", Perception, Vol. 30, 2001, pp. 303-321B.

[20] McCarty, D.P.; Arndt, C.M.; McCabe, S.A. \& D'Amato, D.P. "Effects of compression and individual variability on face recognition performance", Proc. of SPIE, Vol. 5404, 2004, pp. 362-372.

[21] Onifade O.F.W and Adebayo K.J, "Biometric authentication with face recognition using principal component analysis and a feature based technique" In International Journal of Computer Applications (0975 - 8887) pg. 13 - 20, Volume 41- No.1, March 2012

[22] Adebayo Kolawole John, "Combating terrorism with biometric authentication using face recognition" In proc of $10^{\text {th }}$ international conference of the Nigeria Computer Society, 2011. Available online at www.ncs.org.

[23] Adebayo Kolawole John and Onifade Olufade: "Employing Fuzzy Histogram equalization to combat illumination invariance in face recognition systems". In international journal of Intelligent Systems and Applications, Singapore. Vol. 4, No 9, Pg. 54-60, June 2012. Available online at www.mec-press.org.

[24] Haiping Lu, K. N. Plataniotis and A. N. Venetsanopoulos: Uncorrelated Multilinear Principal Component Analysis for Unsupervised Multilinear Subspace Learning, in IEEE transaction on Neural Network, TNN-2009-P-1186.R1, August 2009.

[25] Jin Wang a, Armando Barreto a, Lu Wang a, Yu Chen a, Naphtali Rishe b, Jean Andrian a, Malek Adjouadi: Multilinear principal component analysis for face recognition with fewer features, Elsevier journal of neuron computing Neuron computing Vol 73 (2010) 1550-1555. Available online at www.elsevier.com/locate/neucom.

[26] Richard E woods and Stephen L Eddins: Digital image processing using MATLAB. Dec 1998.

[27] Eswara Reddy and Venkata Narayan: a lossless image compression using traditional and lifting based wavelets. In Signal \& Image Processing: An International Journal (SIPIJ) Vol.3, No.2, April 2012.

[28] Y.W. Chen, "Vector Quantization by principal component analysis", M.S. Thesis, National Tsing Hua University, June, 1998.

[29] H.S.Chu, "A very fast fractal compression algorithm", M.S. Thesis, National Tsing Hua University, June, 1997.

[30] A.E. Jacquin, "Image coding based on a fractal theory of iterated contractive image 
transformations". IEEE Trans on image processing, Vol 1, pg 18-30, 1992.

[31] Rao, K. R., Yip, P., "Discrete Cosine Transforms Algorithms, Advantages, Applications", Academic Press, 1990.

[32] Wallace, G. K. "The JPEG Still Picture Compression Standard", Comm. ACM, vol. 34, no. 4, April 1991, pp. 30- 44.

[33] Belhumeur Peter N., Hespanha Joao P., Kriegman David J.:Eigenfaces vs. Fisherfaces: Recognition Using Class Specific Linear Projection, EuropeanConferenceonComputerVision, 1996.

[34] Etemad Kamran, Chellappa Rama: Discriminant Analysis for Recognition of Human Face Images, Optical Society of America, Volume 14, Number 8, 1997.

[35] Wiskott Laurenz, Fellous Jean-Marc, Kruger Norbert, Malsburg Christoph: Face Recognition by Elastic Bunch Graph Matching, Intelligent Biometric Techniques in Fingerprint and Face Recognition, Chapter 11, pp.355-396, 1999.

[36] Moghaddam Baback, Jebara Tony, Pentland Alex: Bayesian Face Recognition, Pattern Recognition, Vol. 33, No. 11, pps. 1771-1782, 2000.

[37] Bartlett Marian Stewart: Face recognition by Independent Component Analysis, IEEE Transaction son Neural Networks, Volume13, Number 6, 2002.

[38] Li Yun-feng: A Face Recognition System Using Support Vector Machines and Elastic Graph Matching, International Conference on Artificial Intelligence and Computational Intelligence, 2009.

[39] Chai Zhi, Ma Kai-Kuang, Liu Zheng guang: Complex Wavelet-based Face Recognition Using Independent Component Analysis, Fifth International Conference on Intelligent Information Hiding and Multimedia Signal Processing, 2009

[40] Wei Li Xian, Sheng Yang, Qi Wang, Ming Li: Face Recognition Based on Wavelet Transform and PCA, Pacific-Asia Conference on Knowledge Engineering and Software Engineering, 2009.

[41] Bruce A. Draper, Kyungim Baek, Marian Stewart Bartlett and J. Ross Beveridge: Recognizing faces with PCA and ICA, In Computer Vision and Image Understanding 91 (2003) 115-137.

[42] Petra Koruga, Jurica Ševa, Miroslav Bača: A Review of Face Recognition Algorithms and Their Application in Age Estimation. 2012.

[43] A. Hossein Sahoo lizadeh, B. Zargham Heidari, and C. Hamid Dehghani: A New Face Recognition Method using PCA, LDA and Neural Network, in International Journal of Electrical and Electronics Engineering, 2008.

[44] D. Cai, X. He, and J. Han. Semi-supervised discriminant analysis. Computer Vision. IEEE 11th International Conference on Computer Vision, 14:1-7, 2007.

[45] Adebayo Kolawole John and Onifade Olufade Williams: Performance Evaluation of Image Compression on PCA-Based Face Recognition Systems, In IEEE $201212^{\text {th }}$ International Conference on Hybrid Intelligent Systems (HIS), Cochin, India.

[46] Michael W. Marcellin, Michael J. Gormish, Ali Bilgin amd Martin P. Boliek: An Overview of JPEG-2000. In Proc. of IEEE Data Compression Conference, pp. 523-541, 2000.

Adebayo Kolawole John, male, Ijebu-Ode, Nigeria, Lecturer, Ph.D. Scholar., his research directions include Intelligent systems, Biometrics system, Network security, computer vision and Image Processing.

Onifade Williams (Ph.D), male, Ibadan, Nigeria. Senior Lecturer. His research directions include Soft Computing, Fuzzy Systems, intelligence computation and optimal control.

Adekoya Adewale M, male, Ijebu-Ode, Nigeria. Senior Lecturer. His research directions include new Time Series Analysis, Soft Computing and Fuzzy Systems.

How to cite this paper: Adebayo Kolawole John, Onifade Olufade Williams, Adekoya Adewale M.,"Evaluating the Effect of JPEG and JPEG2000 on Selected Face Recognition Algorithms", IJMECS, vol.6, no.1, pp.41-52, 2014.DOI: 10.5815/ijmecs.2014.01.05 